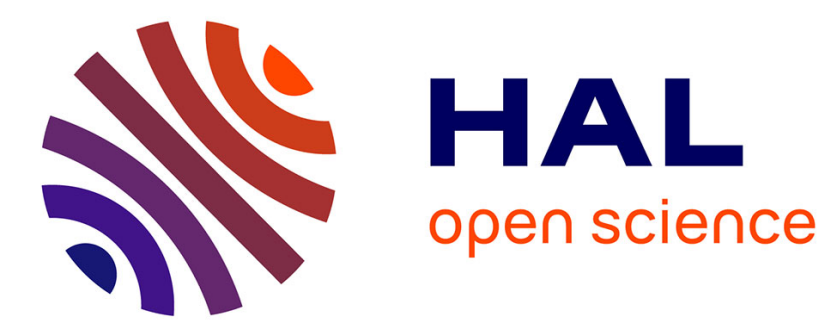

\title{
ARABISME, NATIONALISME ARABE ET IDENTIFICATIONS TRANSNATIONALES ARABES AU 20E SIĖCLE
}

Leyla Dakhli

\section{> To cite this version:}

Leyla Dakhli. ARABISME, NATIONALISME ARABE ET IDENTIFICATIONS TRANSNATIONALES ARABES AU 20E SIÈCLE. Vingtième siècle. Revue d'histoire, 2009, Proche Orient: Foyers, frontières et fractures, 103, 10.3917/ving.103.0012 . hal-01325406

\section{HAL Id: hal-01325406 https://hal.science/hal-01325406}

Submitted on 2 Jun 2016

HAL is a multi-disciplinary open access archive for the deposit and dissemination of scientific research documents, whether they are published or not. The documents may come from teaching and research institutions in France or abroad, or from public or private research centers.
L'archive ouverte pluridisciplinaire $\mathbf{H A L}$, est destinée au dépôt et à la diffusion de documents scientifiques de niveau recherche, publiés ou non, émanant des établissements d'enseignement et de recherche français ou étrangers, des laboratoires publics ou privés. 


\section{ARABISME, NATIONALISME ARABE ET IDENTIFICATIONS TRANSNATIONALES ARABES AU 20E SIÈCLE}

\section{Leyla Dakhli}

\section{Presses de Sciences Po (P.F.N.S.P.) | « Vingtième Siècle. Revue d'histoire »}

$2009 / 3 n^{\circ} 103 \mid$ pages 12 à 25

ISSN 0294-1759

ISBN 9782724631340

Article disponible en ligne à l'adresse :

http://www.cairn.info/revue-vingtieme-siecle-revue-d-histoire-2009-3-page-12.htm

\section{!Pour citer cet article :}

Leyla Dakhli, « Arabisme, nationalisme arabe et identifications transnationales arabes au 20e siècle », Vingtième Siècle. Revue d'histoire 2009/3 (n 103), p. 12-25.

DOI 10.3917/ving.103.0012

Distribution électronique Cairn.info pour Presses de Sciences Po (P.F.N.S.P.).

(C) Presses de Sciences Po (P.F.N.S.P.). Tous droits réservés pour tous pays.

La reproduction ou représentation de cet article, notamment par photocopie, n'est autorisée que dans les limites des conditions générales d'utilisation du site ou, le cas échéant, des conditions générales de la licence souscrite par votre établissement. Toute autre reproduction ou représentation, en tout ou partie, sous quelque forme et de quelque manière que ce soit, est interdite sauf accord préalable et écrit de l'éditeur, en dehors des cas prévus par la législation en vigueur en France. Il est précisé que son stockage dans une base de données est également interdit. 


\section{Foyers}

Contrairement à ce que la fureur médiatique pourrait laisser croire, l'histoire du Proche-Orient contemporain n'est pas un simple agrégat désarticulé de crises et de batailles, une mosaïque illisible de communautés religieuses et de crispations identitaires : elle est au contraire structurée par trois grands foyers idéologiques donnant sa cohérence au siècle qui s'achève. En rendant compte notamment de la dimension éminemment culturelle de l'utopie panarabe, de la complexité et de la plasticité des projets sionistes successifs, de la prégnance de l'héritage impérialiste ottoman au sein du nationalisme turc actuel, ce premier volet offre un premier cadre d'analyse à l'histoire contemporaine du Proche-Orient, à l'histoire de ses grandes utopies politiques et à celle de leur crise actuelle. 


\title{
Arabisme, nationalisme arabe et identifications transnationales arabes au $20^{\mathrm{e}}$ siècle
}

\author{
Leyla Dakhli
}

Région morcelée par les conflits et par des frontières souvent contestées, le MoyenOrient arabe est aussi un espace où le sentiment d'appartenance à un même monde est particulièrement prégnant. Cette impression, née d'une parenté linguistique, historique et religieuse et nourrie par la circulation des personnes, la diffusion d'une même culture populaire et l'existence de médias transnationaux, $s^{\prime}$ est concrétisée au $20^{\mathrm{e}}$ siècle dans deux foyers idéologiques structurants : l'arabisme culturel et le nationalisme arabe. Leyla Dakhli explore ici les articulations et tensions entre culture et politique ainsi que les différentes facettes du rêve d'unité du monde arabe.

Parler du nationalisme arabe ou de l'arabisme revient à déborder les frontières des États nations envisagés dans ce numéro et, dans le même temps, les inclure dans un ensemble plus vaste qui va du Maroc à l'Irak, pour, enfin, les distinguer entre eux : par territoires, par dialectes, par provinces, par strates chronologiques ${ }^{1}$. Apparaît d'abord la carte des pays membres de la Ligue des États arabes, fondée en mars 1945 à Alexandrie. Une ceinture au sud de la Méditerranée et un cœur à l'est. Ici figure la projection d'un rêve politique, mais aussi le lieu de négociations et de tensions au fil du $20^{\mathrm{e}}$

(I) Voir l'article de Philippe Bourmaud dans ce numéro. siècle. Ici figure aussi une «parenté », difficile à contester mais toujours fragile, à fleur de peau, lorsque sont abordées les questions d'appartenance. Car dans la nation arabe, il y a des langues différentes (toutes cousines lorsqu'elles sont arabes, de parenté plus complexe pour le syriaque, l'arménien ou l'araméen, le plus souvent enrichies d'apports multiples venus de tous ceux qui passèrent par ces terres...) ; il y a des filiations historiques plus ou moins continues et toujours multiples, des pharaons aux Phéniciens et aux Carthaginois, en passant par les civilisations qui se sont succédées en Mésopotamie.

La complexité de ce territoire ne se résume donc pas à une période, par ailleurs fort brève, de proclamation d'une unité nationale arabe, favorisée par la désignation d'ennemis à affronter (les États impérialistes européens, l'État d'Israël) et par l'effrondrement d'autres cadres de références (l'Empire ottoman, le califat, la Méditerranée des échelles du Levant, etc.). C'est plutôt un moment du $20^{\mathrm{e}}$ siècle, auquel on peut trouver des définitions mouvantes et complexes, des origines, et peut-être une fin. Ce que l'on appelle le nationalisme arabe, et dont il convient de situer l'âge d'or dans les années 1960, a, selon l'expression de Henry Laurens, « toujours été en crise $^{2}$ ». Mais le sentiment,

(2) Entretien avec Henry Laurens, 4 septembre 2008. Cet article doit beaucoup aux remarques suggestives et à la science de Henry Laurens, qu'il en soit ici remercié. 
de Tanger à Damas ou à Bagdad, de faire partie du «même monde », la possibilité de se comprendre, de partager les mêmes références culturelles est indéniable, même s'il n'est ni une essence ni un acquis inconstestable de la « conquête arabe ». La tension réside en son sein même, quand il s'agit de qualifier à la fois un projet politique, et un sentiment politique, le nationalisme arabe (al-qawmiyya al-'arabiyya), et culturel, l'arabisme. En effet, s'il est relativement aisé de faire l'histoire du projet politique, de ses diverses formes et de son échec, il est plus difficile de restituer au sentiment qu'ont les Arabes de faire partie d'une même nation son origine, sa complexité et sa pérennité. Le nationalisme stricto sensu influe de manière complexe sur la possibilité commune de se sentir partie d'un même ensemble : le monde arabe est déchiré de conflits (Irak-Koweit, guerres civiles, etc.), il est également et surtout fortement mis en question par la tension qui pèse sur son « cœur » moyen-oriental.

\section{Le nationalisme arabe}

Le projet politique de nation arabe a une origine culturelle.La terminologie utilisée par les tenants d'une union politique a mis plusieurs décennies à se fixer réellement. Les mots, comme le corpus théorique, ont longtemps hésité entre plusieurs solidarités possibles. Celles de la langue et de la culture, premières, que l'on rend en français par le vocable « arabisme », semblaient être trop évidentes, pas assez volontaristes pour un projet politique radicalement nouveau. La solidarité islamique désignée en arabe par le vocable umma (communauté ou nation musulmane) ${ }^{1}$,

(I) « Le mot umma, étymologiquement, réfère à la mère (umm), à la tente (bayt, en hébreu olêl et en arabe $a b l$ ) de l'épouse du chef, à la maisonnée du chef, à sa famille cognatique et à sa clientèle ; par opposition à son clan agnatique, et à sa race, désignée, comme Ibn Khaldoun l'a noté, par le mot sha'b, "peuple" ; le sentiment raciste (shu'ûbiyya), l'esprit de corps ('asabiyya) du clan désigne ce particularisme nationaliste. » (Louis Massignon, « L'Umma et ses synonymes » [1946], in Opera Minora, Beyrouth, Dar al-Maaref, 1963, vol. 1, p. 97-103) évidente même pour les non musulmans, imposant de marcher sur les traces d'un empire glorieux en son âge d'or et présent jusqu'à l'abolition du califat en 1924, charriait encore, au moment de l'émergence du nationalisme arabe, trop de mauvais souvenirs ou de faux espoirs. L'incarnation turque rendait impossible le califat arabe, source de débats nombreux parmi les savants et, une fois l'Empire ottoman défunt, il était temps d'inventer autre chose ${ }^{2}$. Néanmoins, alors que se définit une idéologie politique nationale arabe dont on connaît la force et la capacité de mobilisation, les hésitations persistent : les congrès musulmans s'ajoutent aux unions arabes et les journaux célèbrent une nation immémorielle. À mon sens, l'arabisme culturel, loin de constituer simplement un « protonationalisme arabe » comme on le lit souvent (le culturel préparant alors le politique), constitue le socle de cette idéologie et survit au-delà de ses incarnations idéologiques ${ }^{3}$.

\section{Une mission nationale}

Si l'on s'attache à comprendre la genèse d'un projet politique nationaliste arabe, il faut en trouver les racines dans les combats menés au sein des provinces arabes de l'Empire ottoman pour définir une « arabité » et en faire une arme d'émancipation ${ }^{4}$. À l'origine de ce pro-

(2) Voir l'article de Marc Aymes dans ce numéro.

(3) Sur l'arabisme considéré comme un protonationalisme, Ernest Dawn, « The Origins of Arab Nationalism », in Rashid Khalidi, Lisa Anderson, Muhammad Muslih et Reeva S. Simon (dir.), The Origins of Arab Nationalism, New York, Columbia University Press, 1991, p. 3-30.

(4) Une littérature importante est consacrée au nationalisme arabe et à l'arabisme. Voir Rashid Khalidi, Lisa Anderson, Muhammad Muslih et Reeva S. Simon (dir.), op. cit. ; James Jankowski et Israël Gershoni (dir.), Retbinking Nationalism in the Arab Middle East, New York, Columbia University Press, 1997 ; Youssef M. Choueiri, Arab Nationalism : A History, Oxford, Blackwell, 2000 ; William Cleveland, The Making of an Arab Nationalist: Ottomanism and Arabism in the Life and Thought of Sâti' al-Husrî, Princeton, Princeton University Press, 1971 ; Ernest Dawn, From Ottomanism to Arabism : Essays on the Origin of Arab Nationalism, Urbana, University of Illinois Press, 1973 ; Henry Laurens, L'Orient arabe : arabisme et islamisme de 1798 à 1945, Paris, Armand Colin, 1993. 
jet national, il y a donc un travail culturel et intellectuel. Cette histoire s'écrit dans les théories développées à partir du milieu du $19^{\mathrm{e}}$ siècle par les penseurs de la $n a b d a^{1}$, ce mouvement romantique de redécouverte des anciens, de traduction des contemporains, de retour à la critique et au commentaire religieux, ambitionnant de redonner d'abord à la langue arabe sa modernité, sa capacité à dire le monde moderne, tout en l'unifiant. En effet, la grande affaire de ce que l'on appelle la Renaissance arabe fut une affaire de langue. Le projet des réformateurs de l'époque se comprend comme un mouvement double de retour aux origines, de reprise des textes classiques, de republication et de relecture de la littérature arabe ancienne, et de projection vers une modernité incarnée dans la science et dans la littérature occidentale de l'époque. Cette dualité n'est pas univoque. En effet, renouer avec l'adab (c'està-dire avec une culture à prétention universelle mêlant philosophie, morale, littérature et science), est aussi renouer avec la science et la faire entrer à nouveau de plain-pied dans l'humanisme arabe. En mettant en relief ces deux

(I) Sur la nabda, qui signifie réveil en arabe et désigne communément la renaissance littéraire et culturelle débutant au milieu du $19^{\mathrm{e}}$ siècle, la référence reste l'ouvrage classique d'Albert Hourani, Arabic Thought in the Liberal Age, Oxford, Oxford University Press, 1970 ; trad. fr., id., La Pensée arabe et l'Occident, trad. de l'angl. par Sylvie Besse-Ricord, Beyrouth, Naufal, 1983. Certaines études plus récentes sont revenues sur cette période pour en faire l'histoire sociale et intellectuelle, notamment pour y découvrir que les artisans de cette nabda étaient aussi des femmes, des paysans, des ouvriers. Ils ont également confronté la pensée de la nabda avec les politiques concrètes mises en œuvre par leurs zélateurs. Voir les contributions de l'ouvrage : Dyala Hamzah (dir.), The Arab Intellectual and the Question of Modernity, Londres, Routledge, 2009 ; Michael Gasper, The Power of Representation. Publics, Peasants, and Islam in Egypt, Stanford, Stanford University Press, 2009 ; Jens Hanssen, Fin de Siècle Beirut : The Making of an Ottoman Provincial Capital, Oxford, Clarendon Press, 2005 ; Orit Bashkin, The Other Iraq : Pluralism and Culture in Hashemite Iraq, Stanford, Stanford University Press, 2009.

(2) L'adab est la culture de l'honnête homme, c'est-à-dire un mélange de science, de littérature et de raffinement dans les mœurs. Cela signifie, par extension, les bonnes manières. pôles de la renaissance, l'un intérieur et historique, l'autre extérieur et se projetant vers une modernité, on comprend d'emblée le projet de la nabda comme lié à une mobilité forte. Elle est d'abord une œuvre de passage, de traduction ${ }^{3}$. Ainsi les grands auteurs de l'époque sont-ils souvent des traducteurs, des passeurs, des adaptateurs ${ }^{4}$. Ce passage a souvent été résumé dans une mise en relief de l'emprunt comme moteur de la nabda. En ce qui concerne le nationalisme, l'arabisme serait alors une idée « importée », européenne, élaborée notamment dans les universités missionnaires de la région ${ }^{5}$. Cette idée est battue en brèche par des travaux récents qui montrent une réalité plus complexe $^{6}$. Comme l'écrit l'historien Zachary Lockman à propos de l'histoire de l'Égypte du début du $20^{\mathrm{e}}$ siècle :

« La question dominant cette histoire intellectuelle [traditionnelle] est celle de savoir quel rapport les membres de l'élite égyptienne entretenaient avec les concepts et les idées nées en Europe occidentale, et comment ils les adoptaient éventuellement entièrement ou en partie. Ce processus est considéré comme le catalyseur clé du changement social : des membres d'une élite éduquée rencontrent certaines idées originalement étrangères, essaient de leur donner sens dans leur propres présupposés culturels, et enfin les mettent en œuvre dans leur propre société, à travers leurs écrits, ou les incarnent dans des institutions nouvelles ou "réformées",

(3) Voir l'article d'Orit Bashkin dans ce numéro.

(4) Pour un exemple de circulation des idées (socialistes), voir Ilham Khoury-Makdisi, « Intellectuels, militants et travailleurs : la construction de la gauche en Égypte, 1870 1914 », Cabiers d'bistoire. Revue d'histoire critique, 105-106, juillet-décembre 2008 .

(5) C'est le sens du titre français de l'ouvrage d'Albert Hourani, La Pensée arabe et l'Occident (op. cit.), qui rend justice à une partie de l'œuvre. C'est aussi la perspective choisie dans la plupart des histoires littéraires classiques de la région.

(6) Sur l'arabisme, voir Ernest Dawn, op. cit. ; sur les missionnaires, voir Ussama Makdisi, Artillery of Heaven : American Missionaries and the Failed Conversion of the Middle East, Cornell, Cornell University Press, 2008. 
en faisant des moyens pour atteindre les classes les plus basses ${ }^{1}$.

Pour en finir avec cette vision de l'histoire qui fait de la réforme et de l'adaptation la clé de compréhension des sociétés de cette région, il serait plus juste de parler de circulation, de recourir à la notion de cosmopolitisme des élites pour comprendre comment les intellectuels, souvent multilingues, s'approprient, très naturellement, les cultures qu'ils traversent et sont eux-mêmes traversés par les idées de leur temps.

Ce n'est que par la suite que le nationalisme arabe prend une tonalité plus politique. Le monde arabe est alors partagé entre les puissances coloniales européennes et l'Empire ottoman. Ses littérateurs et intellectuels sont dispersés dans des provinces où ils trouvent refuge pour contester les pouvoirs en place. Des lieux comme Le Caire, mais aussi des capitales européennes et américaines deviennent le centre d'élaboration de projets nationalistes multiples. Les provinces arabes de l'Empire s'émancipent à la faveur de la proclamation d'une Constitution libérale en 1876 ; mais d'autres mouvements réformateurs accueillent également les prémices de cette pensée nationaliste arabe, comme la Tunisie de Kheireddine Pacha, juste avant l'installation du protectorat français ${ }^{2}$.

Ce projet trouve une forme d'achèvement dans l'éclatement de l'Empire ottoman, la naissance d'un certain nombre d'États arabes et la création des Académies de langue arabe (Le

(I) Zachary Lockman, « Explorations in the Field : Lost Voices and Emerging Practices in Egypt, 1882-1914 », in Israel Gershoni, Hakan Erdem et Ursula Wokoeck (dir.), Histories of the Modern Middle East : New Directions, Boulder, Lynn Rienner Publishers, 2002.

(2) Kheireddine Pacha (ou khayr ad-dîn al-tûnsî) est un homme politique et réformateur (1822-1890), auteur d'un ouvrage célèbre : Kitâb akram al-masâlik fî ma'rifati abwâl almamâlik [Le plus sûr moyen pour connaître l'état des nations], Tunis, 1867.
Caire, Damas) $)^{3}$. Il trouve aussi une expression concrète et politique, d'abord au sein de l'Empire à travers le parti ottoman de la décentralisation ${ }^{4}$ puis, au fil des combats, dans la « révolte arabe » de 1916. Auparavant, la révolution constitutionnelle de 1908 avait permis l'élection de députés, œuvrant, selon leurs propres mots, à «donner voix aux Arabes » au sein de l'Empire ${ }^{5}$. L'arabisme, tel qu'on commence à le nommer, n'est pas encore un « panarabisme », ou un nationalisme arabe. Imaginé dans le cadre de la révolte comme un soulèvement des provinces arabes, il se recentre par le jeux des ambitions impériales sur des États, qui ne sont pas des États nations, mais des États territoires, dont les contours ont été définis par d'autres dans le règlement du premier conflit mondial. Par delà ces cadres coloniaux ou postcoloniaux, des unités plus larges sont revendiquées, définissant des territoires nationaux mouvants et des ambitions dynastiques (Grande Syrie des nationalistes syriens, croissant fertile des Hachémites, Grand Liban maronite, etc.).

\section{Une idéologie révolutionnaire}

Ce n'est qu'après la deuxième guerre mondiale qu'émerge un nationalisme arabe comme projet politique concret, incarné par des partis politiques et des leaders. Il est forgé dans les années de l'entre-deux-guerres, au sein d'un milieu intellectuel qui porte l'héritage de la $n a b d a$, mais le transforme dans un sens plus politique, moins nostalgique, plus tourné vers l'action et vers l'avenir.

(3) Je me permets de renvoyer à Leyla Dakhli, Une génération d'intellectuels arabes : Liban, Syrie, 1908-1940, Paris, Karthala, 2009. Voir le compte rendu dans ce numéro (p. 209-211).

(4) Créée en 1912 au Caire, cette association regroupe des personnalités d'horizons politiques très différents, aussi bien des défenseurs de la représentation des provinces arabes au sein de l'Empire que des tenants de l'autonomie du Mont-Liban. Elle reste dans les limites de l'application de la loi ottomane.

(5) Fāris al-Khūrī, Awrāq Fāris al-Khūrì [Papiers Fāris al-Khūrī], Damas, Dār Tlas, 1989, vol. 1, p. 92-93. 
Les hommes et les femmes qui font littéralement entrer en politique l'arabisme le font dans un contexte impérialiste, celui d'une tutelle coloniale française ou britannique, imposée en règlement de la première guerre mondiale et largement perçue comme une félonie. Il s'allie alors d'emblée avec des mouvements nationalistes plus populaires, voire populistes, qui s'organisent pour lutter contre le nouveau pouvoir et trouver leur place dans le nouveau rapport de force ${ }^{1}$. Ils entrent en politique également dans un contexte d'accommodement du discours arabiste aux limites du débat politique national (au sens restreint des frontières récemment tracées dans la région).

L'entre-deux-guerres définit les limites des notions complémentaires d'arabisme, de nationalisme, de nationalisme arabe et de panarabisme ; ces différentes appartenances trouvent leur ancrage dans le règlement du premier conflit mondial, non sans heurts et sans contestation, alimentant des frustrations (le Grand Liban ou la Grande Syrie), des irrédentismes (le Sandjak d'Alexandrette que se disputent nationalistes turcs et syriens), des nostalgies (le Croissant fertile rêvé par la monarchie hachémite et le colonel Lawrence).

Cette évolution peut se voir par exemple chez les rédacteurs du journal damascène al-Talî'a, journal de « l'avant-garde » au tout début des années 1930 ; leur projet nationaliste est d'emblée révolutionnaire, intrinsèquement socialiste, et s'incarne nécessairement dans une nouvelle génération qui critique l'œuvre de ses pères, corrompus et incapables de faire accéder à l'indépendance réelle. S'il est plus nettement idéologique, le projet nationaliste arabe s'appuie alors sur une vision culturelle de la nation. Il est aussi d'emblée hors du répertoire

(I) Voir James Gelvin, Divided Loyalties : Nationalism and Mass Politics in Syria at the Close of Empire, Berkeley, University of California Press, 1998. laïc. L'islam, dans la pensée de Michel Aflaq, l'un des collaborateurs du journal et père fondateur du parti Ba'th, est la première expression du génie arabe, une des premières incarnations du projet national : « Le nationalisme arabe n'est pas une théorie, mais la source de toute théorie. Ce n'est pas non plus le résultat d'une pensée pure, mais la mère de toute pensée. Au lieu de réduire en esclavage l'art, il est sa source et son âme ${ }^{2}$. »

Le nationalisme arabe est alors élaboré comme une profession de foi. Comme l'écrit Youssef Choueiri : « [Aflaq] insista de nombreuses fois sur le fait que ce qu'il avait à dire était plus un point de vue [nadbra] qu'un système [nadhariyya]. Néanmoins, la terminologie qu'il inventa et ses discours furent intégrés dans le discours politique d'une génération arabe entière ${ }^{3}$. »C C'est dans le passage de la théorie, voire de la foi, à une forme jusque-là inconnue de mobilisation populaire que réside le « mystère ».

L'entre-deux-guerres est le moment où les théorisations entrent en dialogue les unes avec les autres. Les arabistes de la génération précédente, ayant vu la fin de l'Empire ottoman comme une opportunité de trouver enfin leur place dans les États en constitution, entrent en politique et négocient pour eux-mêmes un territoire, un public, un espace public. Le personnel politique libanais, syrien, irakien ou palestinien fait ses premières armes en défendant des solidarités, des cohérences territoriales, des clientèles. Il utilise le nationalisme arabe comme une carte à jouer contre les puissances mandataires (et le projet sioniste) et dans les négociations avec les autres États arabes. Certes, les frontières sont encore poreuses et le personnel politique syrien, par exemple,

(2) Michel Aflaq, Fî sabîl al-ba'th [Pour le parti de la résurrection], Beyrouth, Dar al-Talî'a, 1963, p. 41.

(3) Youssef M. Choueiri, op. cit., p. 154 
est constitué d'hommes qui sont parfois stricto sensu des Palestiniens ou des Libanais ; plus encore, l'Irak hachémite, héritier de la révolte arabe de 1916 et du Royaume arabe de Damas de 1918-1920, attire nombre d'intellectuels et d'hommes d'armes de l'ensemble de la région. Mais, pour l'essentiel, les espaces délimités par les traités servent de cadre pour les carrières politiques de ces hommes.

Parallèlement, une génération nouvelle, plus radicale, moins encombrée par le souvenir de l'Empire, pose les fondements d'un « nationalisme arabe transnational », structuré autour de partis comme le Ba'th ou les Frères musulmans. Ce mouvement, multiforme et complexe, trouve son expansion dans les années 1940.

Il convient de lier l'essor du projet nationaliste arabe, qui ne se limite pas aux théorisations de Michel Aflaq ${ }^{1}$, Zaki al-Arsuzi ${ }^{2}$, Sâti' al-Husrî̀' ou 'Abd al-Rahmân Shahbandar',

(I) Michel Aflaq (1910-1989) est l'un des fondateurs du parti Ba'th. Il est né à Damas dans une famille grecque-orthodoxe. Il fait des études de lettres à Paris, où il rencontre l'autre fondateur du parti, le musulman sunnite Salah al-Dîn al-Bîtâr. Dans l'entre-deux-guerres, il sont tout deux des contributeurs réguliers des journaux et revues damascènes, avant de fonder le parti de la résurrection arabe (Ba'th) en 1943.

(2) Zaki al-Arsuzi (1899-1966) est considéré comme le troisième fondateur du parti Ba'th. Né à Lattakié dans une famille alaouite, il a également fait ses études à Paris. Il commence à théoriser son nationalisme arabe dans l'entre-deux-guerres, en même temps qu'il enseigne dans la région d'Alep. Il fonde, dans la deuxième moitié dans années 1930, la Ligue d'action nationale. Il rejoint le Ba'th à partir de 1944-1945. Voir Keith D. Watenpaugh, « Creating Phantoms : Zaki al-Arsuzi, the Alexandretta Crisis and the Formation of Modern Arab Nationalism in Syria », The International fournal of Middle East Studies, 28, 1996, p. 363-389.

(3) Sâti' al-Husrî (1879-1967) est un théoricien du nationalisme arabe. Responsable des programmes aux ministères de l'Éducation aussi bien en Irak où il suit l'émir Faysal en 1920 qu'en Syrie à partir de 1943, il est considéré comme le principal artisan de la diffusion de la vulgate ba'thiste et nationaliste arabe. Voir Maher Charif, Rihanat al-nabda fi'l-fikr al-'arabi, Damas, Dar al-Mada, 2000.

(4) 'Abd al-Rahmân Shahbandar (1880-1940) est l'un des leaders de la révolte syrienne contre le mandat français, en 1920 comme en 1925, au sein du parti du peuple (al-Sha'b). Il est exilé en Égypte de 1926 à 1937. À son retour en Syrie, critiquant peut-être trop ouvertement la politique de « collaboration »du à son incarnation dans une vie parlementaire active, à la fois dans le cadre des protectorats et mandats et bien plus encore au sein d'États nouvellement indépendants. Dans la lecture faite aujourd'hui de ces questions, on a trop souvent tendance à lire le devenir autoritaire des régimes arabes comme une donnée inéluctable : il n'en est rien. Les luttes d'émancipation nationales sont les mises en acte de ces théories révolutionnaires, en particulier la lutte algérienne, pour laquelle l'ensemble du monde arabe se mobilise et s'enthousiasme. Le nationalisme trouve aussi des voix dans le mouvement national libanais, comme dans une partie du mouvement national palestinien.

Mais c'est lorsqu'il prend le visage du nassérisme que le nationalisme arabe s'incarne de la manière la plus immédiate. Il coïncide avec le discours d'un homme et sa popularité jamais égalée, mais aussi avec un mouvement culturel et artistique qui met l'Égypte, ses chanteurs, ses stars de cinéma, ses paysages mêmes, au centre des « rêves arabes ».

Pourtant, à peine brandi, cet étendard, fortement attaché à l'idée de révolution qui lui devient corollaire, confronté à l'évolution des régimes nationaux et de la situation régionale, s'achève. On peut considérer que la mort du nationalisme arabe est déjà présent au sommet de la Ligue arabe de 1964, lorsque les États arabes réunis abandonnent ipso facto le projet unitaire. On peut aussi considérer que l'assassinat de Kamal Djoumblatt en $1977^{5}$, au Liban, marque le chant de cygne du projet.

gouvernement syrien, il est assassiné en 1940. Voir Leyla Dakhli, op. cit., chap. 5 .

(5) Kamal Djoumblatt (1917-1977), leader druze de la montagne libanaise, était également un des chefs de la gauche arabe et libanaise à travers le parti socialiste progressiste. Sur cette question, je renvoie à l'article de Dima de Clerck dans le présent volume. Elle montre bien comment l'appartenance panarabe, ou même simplement socialiste, a été mise à mal par la guerre civile ; le Liban est alors simplement une vision à grande échelle de ce qui est à l'œuvre dans l'ensemble du monde arabe, faute de véritable ciment national ou idéologique. 


\section{Des incarnations autoritaires}

Dans le même temps, le nationalisme arabe s'est peu à peu incarné et figé dans des régimes autoritaires qui ont confisqué cette idéologie et en ont fait une arme pour combattre toute opposition, qu'elle soit communiste ou islamiste. Au fil des années 1960, après la fin des expériences démocratiques de l'après-guerre (notamment en Syrie, au Liban, en Irak...), après la fin des luttes anticoloniales (1962), le nationalisme arabe a perdu son potentiel révolutionnaire pour trouver son expression principale dans des régimes de plus en plus durs, toujours en guerre et dont l'unique vecteur « émotionnel » était devenu le président Nasser, leader charismatique autant que dictateur impitoyable. Les différents régimes en place, au nom même de leur idéologie, créèrent des armées de masse, contribuant à encadrer la nation et à éviter les possibilités de coups d'État comme de soulèvements. Ces nouveaux cadres militaires étaient peu à peu mis au service du projet et devenaient des privilégiés à qui l'on confiait non seulement la défense du pays et la guerre extérieure, mais aussi des industries, des carrières, des rentes de situation.

La fin des expériences démocratiques et parlementaires est également responsable de l'impossibilité dans laquelle se sont trouvés les différents régimes autoritaires arabes à fusionner dans une seule entité politique. Leur maintien au pouvoir ne pouvait pas passer par une telle dilution. Bien au contraire, ils entrèrent en rivalité pour le leadership arabe. L'expérience de la République arabe unie, union entre la Syrie (alors parlementariste quoique secouée de crises et de coups d'États) et l'Égypte (de 1958 à 1960), qui fut l'occasion pour Nasser de marginaliser les autres forces nationalistes arabes (notamment le Ba'th et le parti communiste), en offre l'illustration parfaite ${ }^{1}$.

(I) Sur cette période, encore peu explorée par l'historiographie, je renvoie aux Mémoires de l'un de ses acteurs, Akram
Ces régimes ont pérennisé l'absence de référent démocratique commun. Ils ont pour la plupart effacé le souvenir de l'après-guerre et du pluralisme et se sont maintenus suffisamment longtemps au pouvoir pour que les jeunes générations, très majoritaires, n'aient plus aucun souvenir de ces périodes. Si l'on peut affirmer que le nationalisme arabe fut l'idéologie commune d'une génération, celle des années 1940-1960, elle est peu à peu supplantée par d'autres, et notamment par l'islamisme, investi du potentiel révolutionnaire perdu. Pourtant, il ne s'agit pas d'une substitution d'une idéologie à une autre. Cette affirmation, que l'on rencontre souvent dans les études sur la région expliquant l'essor de l'islamisme par la « dénationalisation $\gg$ ou la fin du projet nationaliste arabe, découle d'une forme de myopie des études sur la région qui, un temps obsédées par le nationalisme arabe dans le contexte de la guerre froide, ne voyaient pas les islamistes pourtant présents dès le milieu des années $1920^{2}$. Islamisme et nationalisme arabe ont partie liée : ces deux idéologies convergent à plus d'une occasion, le nationalisme arabe se teintant souvent de « panislamisme » (l'une des formes de cette convergence est le débat sur le califat arabe, né dans les dernières décennies de l'Empire ottoman et devenu pressant avec la fin du califat, en $1924^{3}$ ), l'islamisme politique déve-

Hourani, député au parlement syrien à de nombreuses reprises et leader du parti socialiste arabe (Akram Hourani, Mudhakkarât Akram al-Hûrânî, Le Caire, Maktabat Madbûlî, 2000). Ces Mémoires ont eu un grand retentissement lors de leur publication (interdite en Syrie), et ont déclenché une véritable « guerre des Mémoires » dans l'édition arabe. Henry Laurens a initié dans le cadre de son séminaire au Collège de France un travail sur ces sources.

(2) À l'exception, notable, d'Olivier Carré, qui travailla dès la fin des années 1970 à la fois sur le nationalisme, le socialisme et l'islamisme dans le monde arabe, en montrant bien les filiations et les passages entre ces différents mouvements et registres. Voir notamment Olivier Carré, Mystique et politique : lecture révolutionnaire du Coran par Sayyid Qutb, frère musulman radical, Paris, Presses de Sciences Po/Ed. du Cerf, 1984.

(3) Voir Henri Laoust, « Le Califat dans la doctrine de Rachid Rida », trad. annotée d'Al-Khilâfa aw al-imâma al-'uzma [Le califat ou l'imamat suprême], Le Caire, 1926, in Mémoires de 
loppant une forme de nationalisme arabe. Les itinéraires politiques des leaders de la région, à commencer par celui des officiers libres et de Nasser, montrent bien la coïncidence possible des deux idéologies. Et l'on peut même considérer que l'islamisme est mort avec le nationalisme arabe, dans la phase de mondialisation qui a accompagné l'essor d'un islam politique radical et transnational.

Enfin, depuis leur accession à l'indépendance, les États arabes ont eu des cheminements propres dans le cadre de frontières parfois subies, mais qui définissaient les limites de l'exercice étatique, administratif, etc. Progressivement, ces limites ont permis de construire des identités, une mémoire commune, un terrain d'affrontement. La Ligue arabe elle-même, ayant abandonné implicitement son ambition unitaire, contribua à créer une coopération entre les États (encore très mal connue et très peu étudiée $^{1}$ ) autour de liens commerciaux, monétaires, culturels, etc. En quatre-vingt-dix ans, chacun a pris sa voie. L'inertie des frontières a imposé un cadre national, aussi contesté qu'il puisse être (guerre entre l'Iran et l'Irak ou invasion du Koweït en 1990). Les aventures militaires communes des États arabes restent celles qui les opposent à l'État d'Israël. L'échec de la guerre de 1967 a ainsi été la fin d'un rêve, certainement, mais cette fin était préparée par de nombreux épisodes. Elle sera confirmée encore, s'il en était besoin, par l'épisode du « Septembre noir $\gg$ et la guerre du Liban. Ce repli sur l'espace national restreint n'a pas affaibli le sentiment d'appartenance commune, socle de l'idéologie nationaliste arabe : al-'urûba.

\section{Al-'urûba, une communauté arabe}

Le terme de «'urûba», qui signifie «le fait d'être arabe », est parfois traduit par un néo-

l'Institut français de Damas, Damas, Institut français de Damas, 1938.

(I) On ne sait pas grand chose, notamment, de l'action des différentes agences de la Ligue arabe. logisme étrange, l'arabité. Il a l'avantage de faire sentir l'aspect qualitatif de cette notion, peu mesurable, difficile à décrire et à cerner. L'arabité est quelque chose d'indéfini, souvent ramenée à la langue, qui en est le vecteur principal, mais qui va bien au-delà d'une compréhension linguistique mutuelle, très relative en fonction des dialectes. La voix des Arabes n'est pas seulement leur langue, elle est aussi une musique, des sujets de préoccupation communs, des questionnements et des émotions.

\section{La « voix des Arabes», d'Om Kalthoum}

à al-fazeera

C'est par le constat de la persistance de l'idée nationale arabe que se poursuit l'exploration de l'arabisme. Le nationalisme arabe a pu n'être qu'une brève parenthèse dans l'histoire de la région. Pourtant, comme l'écrit Rashid Khalidi, « le nationalisme arabe, qui peut se résumer à l'idée que les Arabes sont un peuple uni par des liens spéciaux de langue et d'histoire (et de religion, ajouteraient certains), et que leur organisation politique devrait montrer ces liens d'une manière ou d'une autre, a toujours une force certaine dans le monde arabe ${ }^{2} \gg$.

On peut émettre l'hypothèse que l'abandon même de la perspective de l'unité a permis aux Arabes de donner à leur sentiment d'appartenance commune de nouvelles formes. C'est en tant que « communauté imaginée », pour reprendre l'expression de Benedict Anderson ${ }^{3}$, que le nationalisme arabe trouve son expression la plus juste. Et cette projection continue de s'appliquer, alors même que le projet politique s'affaiblit. L'un des accords les plus marquants entre pays arabes est certainement la Consti-

(2) Rashid Khalidi, introduction à Rashid Khalidi, Lisa Anderson, Muhammad Muslih et Reeva S. Simon, op. cit., p. vii à xix.

(3) Benedict Anderson, Imagined Communities : Reflections on the Origin and Spread of Nationalism, Londres, Verso, 1983, 2006 ; trad. fr., id., L'Imaginaire national : réflexions sur l'origine et l'essor du nationalisme, trad. de l'angl. par Pierre-Emmanuel Dauzat, Paris, La Découverte, 2006. 
tution d'ArabSat en 1976, un satellite commun qui a permis aux chaînes de télévision transnationales de s'installer dans la région. Celles-ci n'ont pas cherché à unifier, mais bien à échanger. Cette histoire a commencé quelques années plus tôt, et, si l'on inclut la presse écrite, elle a débuté au siècle précédent ${ }^{1}$. D'abord, ce sont des programmes égyptiens (et, dans une moindre mesure, libanais) qui inondent le marché arabe aux temps du transistor : Om Kalthoum, 'Abd al-Halim, la radio nassérienne Sawt al-'arab (la voix des Arabes)... sont les voix de l'âge d'or du nationalisme arabe ${ }^{2}$. Elles convergeaient dans un vaste projet, à la fois politique, culturel et médiatique. Comme l'écrit Elias Khoury :

« La Voix des Arabes incarnait vraiment un projet politique grâce à un développement technique, celui de la naissance du transistor, qui allait provoquer un total renversement des données politico-médiatiques en permettant que la voix de Nasser, émise depuis Le Caire, puisse être entendue jusque dans les Aurès en Algérie ${ }^{3}$. »

Aujourd'hui, les voix sont plurielles : la chaîne d'information continue al-fazeera, mais aussi la « Star Academy » arabe, les chaînes de divertissement libanaises, la joute poétique télévisée d'Abu Dhabi $T V$ « Le poète du million », les feuilletons à succès diffusés pendant le Ramadan (feuilleton historique retraçant la vie du roi Farouk ou de Nasser, bleuettes turques doublées en dialecte syrien, etc.).

Loin de rester des spectateurs de cette culture populaire, les hommes et les femmes circulent de plus en plus au sein du monde arabe. Les

(I) Voir Tourya Guaaybess et Yves Gonzalez (dir.), Les Arabes parlent aux Arabes: la révolution de l'information dans le monde arabe, Arles, Sindbad-Actes Sud, 2009.

(2) Voir François Moriconi-Ebrard et Ménar Rateb, « Le Caire métropole du Monde arabe ? Éléments de réflexion », Lettre d'information de l'Observatoire urbain du Caire contemporain, 45, 1996, p. 35-45.

(3) Elias Khoury, « Pour en finir avec la "rue arabe" », $\mathrm{Al}$ Quds al-'arabi, ${ }^{\text {er }}$ septembre 2008 (trad. et prés. sur le blog d'Yves Gonzalez-Quijano, http://cpa.hypotheses.org/353). migrations internes se sont multipliées depuis les années 1980. Les migrations vers le MoyenOrient ou le Golfe ne concernent plus seulement massivement les Orientaux eux-mêmes, mais aussi les Maghrébins (il faudrait établir probablement une corrélation avec la fermeture des frontières européennes), même s'il faut relativiser cette ouverture : alors qu'en 1975, le taux de main-d'œuvre provenant des pays arabes dans les pays du Golfe atteignait $75 \%$ de la main-d'œuvre étrangère, il ne dépasse pas les 19 \% actuellement, à cause de multiples facteurs comme les crises et les guerres ou la fermeture d'un certain nombre de débouchés économiques. La main-d'œuvre arabe est par ailleurs fortement concurrencée par la main-d'œuvre asiatique, peu coûteuse. La rente pétrolière est née dans la période d'essor du nationalisme arabe, et ces migrations interarabes, surtout en direction des États pétroliers, ont fait partie des facteurs de diffusion de l'idée panarabe. Pourtant, tout comme la Ligue arabe a relativement échoué à créer un véritable espace commun, les échanges commerciaux interarabes sont restés assez marginaux face au poids des échanges avec les pays les plus développés, insérant la région dans les circuits de la mondialisation ${ }^{4}$.

Aujourd'hui pourtant, la circulation des hommes et des femmes est plus multiforme. Les frontières sont plus ouvertes et mouvantes que dans les années 1990. Le phénomène de libéralisation des médias a accéléré la circulation des personnes, pour trouver du travail, mais aussi pour découvrir les autres pays. Jusqu'aux années 1980, un monopole étatique s'exerçait encore sur les médias dans toute la région ${ }^{5}$. RMC Lobnan, la $B B C$ et la radio israé-

(4) Voir Leila Vignal, La Mondialisation au Proche-Orient : espaces métropolitains et émergence d'un système régional (Le Caire, Beyrouth, Damas), Avignon, Université d'Avignon et des pays de Vaucluse, 2006.

(5) Voir Tourya Guaaybess, « De l'État-émetteur à l'émetteur État dans le champ télévisuel égyptien », in Franck Mermier (dir.), Mondialisation et nouveaux médias dans l'espace arabe, Paris, Maisonneuve et Larose, 2003, p. 103-123. 
lienne en arabe étaient les sources d'information « libres » que l'on écoutait sur place. Dans les années 1990, on peut dire que les Arabes ont gagné la « bataille de l'information », la guerre du Golfe (1991) devant probablement être considérée comme la fin du monopole de CNN. À cela plusieurs raisons : le professionnalisme d'abord et la qualité du travail des journalistes d'al-fazeera, d'al-'Arabiyya ou de la télévision d'Abu Dhabi (Abu Dhabi TV); les moyens investis qui leur permettent d'avoir un réseau dense de correspondants (et cela jusqu'en Israël) et des journalistes brillants, issus de toutes les régions du monde arabe ; la réactivité forte et l'interaction avec un public extrêmement vaste. Cette bataille a été gagnée sur le terrain d'une information extrêmement normalisée, sur le « format $C N N »$, contrant d'autant plus efficacement cette dernière ${ }^{1}$.

Cette nouvelle circulation et la pluralité des voix de « l'arabisme » remet en question les formes mêmes selon lesquelles les théoriciens du nationalisme arabe ont pensé l'unité arabe : alors qu'ils fabriquaient un arabe moyen (« l'arabe médian », selon l'expression de Jacques Berque) issu du classique censé établir une médiation entre les dialectes, c'est aujourd'hui la prolifération des dialectes qui se lit et s'entend dans les médias (presse écrite populaire, télévions, Internet, etc.) ; le registre de l'émotion ne pouvait pas être atteint dans une langue formelle, censée appartenir à tous mais qui n'appartenait à personne. Nasser lui-même, lorsqu'il voulait parler au cœur des Arabes, et en particulier des Égyptiens, passait régulièrement au dialecte. Ainsi lors de son discours au lendemain de la défaite de 1967, annonçant sa démission, il s'adressa à ses frères sur le ton

(I) Voir Olfa Lamloum, Al-fazira, miroir rebelle et ambigu du monde arabe, Paris, La Découverte, 2004 ; Tourya Gaaybess, Télévisions arabes sur orbite : un système médiatique en mutation (1960-2004), Paris, CNRS éditions, 2005 ; Franck Mermier (dir.), op. cit. de l'intime et fit déferler la foule dans les rues du Caire. Si l'égyptien a été pendant de longues années la langue vernaculaire du divertissement et de la politique, aujourd'hui, le dialecte syro-libanais s'impose peut-être, dans le seul registre de l'entertainment.

\section{Construire une réponse au sionisme?}

Faire ce constat revient à faire celui de l'échec d'un projet politique, mais aussi à revenir sur la manière dont nous, chercheurs en sciences sociales, nous fondons sur l'usage de concepts pour désigner des réalités humaines toujours plus complexes. Les idéologues eux-mêmes transforment leurs discours et leurs stratégies en fonction des sentiments d'appartenance, des registres d'émotion auxquels ils ont recours. Tout comme Nasser, nationaliste arabe qui pouvait aussi jouer sur la fibre religieuse ou sur le nationalisme égyptien, on peut considérer par exemple que le discours et la stratégie politique du Hezbollah et de Hassan Nasrallah pendant l'offensive israélienne de l'été 2006 a relevé du nationalisme arabe plus que du prêche islamiste. Il a ainsi réussi à fédérer les Arabes contre l'invasion israélienne.

En réalité, il faut reconnaître qu'en dehors du registre de l'émotion, de l'évidence de la culture commune, le nationalisme arabe, sous sa forme militante, trouve surtout appui sur l'anti-impérialisme. En cela encore, il rejoint les autres idéologies en place dans la région.

En ce sens, le nationalisme arabe est une réponse, toujours présente même sous une forme moins organisée et plus atténuée, dès que le monde arabe est menacé. On peut considérer que le moment politique du nationalisme arabe prend fin en 1962-1964, parce que disparaît le dernier grand foyer de lutte anticoloniale dans le monde arabe : l'Algérie française. Mais les interventions extérieures dans les affaires moyen-orientales ne cessent de réactiver ce réflexe, sous une forme réelle ou 
fantasmée. Surtout, après la victoire nationaliste en Algérie, le front impérialiste est désormais incarné dans l'État d'Israël et son idéologie sioniste, pays qui se présente comme appartenant au monde occidental et comme le seul État démocratique de la région : cette définition de soi est immédiatement traduite par les voisins arabes dans la langue de l'antiimpérialisme. Comme le fait justement remarquer Henry Laurens, Israël est bien, dans la bouche de Nasser, « la citadelle de l'impérialisme » (qal'a), ou dans le langage ba'thiste, « la base de l'impérialisme » (qẩida). On peut voir dans cette incompréhension occidentale l'une des raisons de l'échec du projet américain de « Grand Moyen-Orient » : on y trouve un usage surabondant du mot « démocratie » au détriment de celui de « nation $»$ ou $\ll$ nationalisme $»$, souvent remplacé par « terrorisme $»^{1}$.

Dans le même temps, les islamistes ont récupéré à leur profit des référents forts de cette lutte anti-impérialiste, auparavant apanage des arabistes : le vocabulaire de la révolution et de la résistance est à présent de leur côté, s'incarnant dans un nouveau soulèvement, transnational et anti-impérial. Dans leur analyse de la lutte à mener contre l'État d'Israël, cette stratégie est nettement marquée. Le sionisme, fondé sur un volontarisme fort, fonctionne comme un repoussoir étranger. Les Frères musulmans seuls ont pu considérer qu'il fallait prendre exemple sur l'action des sionistes, notamment parce qu'il s'agissait d'un nationalisme fondé sur la religion. Les nationalistes arabes, eux, trouvent leur inspiration plutôt dans l'organisation de l'État sioniste ; Muhammad Husayn Haykal rapporte ainsi que Nasser aurait dit à Arafat : «Tu seras mon Irgoun et mon Stern ${ }^{2}$. »

(I) Entretien avec Henry Laurens cité plus haut.

(2) Journaliste et homme politique égyptien, né en 1923, il a accompagné la carrière de Nasser, devenant parfois lui-même la « voix de l'Égypte », notamment via le journal al-Abram. Ses Mémoires ont été publiés et il les a également livrés, sous forme de conversations, à la chaîne de télévision al-fazeera.
Dans ce cas, la cause palestinienne fonctionne comme le bras armé du nationalisme arabe.

La comparaison s'arrête là. Le nationalisme arabe, vécu comme une évidence, n'a pas besoin du volontarisme déployé par les sionistes. Il est sans cesse alimenté par un patrimoine, une circulation, des productions nouvelles. On pourrait dire en simplifiant que le tarab, l'émotion toute particulière produite par l'écoute musicale, est ce qui fonde l'arabité. Si cette vision émotionnelle se heurte à celle de l'État et à l'absence de démocratie, elle fonctionne exactement à l'inverse du sionisme, où le projet commande tout ${ }^{3}$.

En retour, le conflit israélo-arabe a participé à construire de nouveaux jalons dans la définition du nationalisme arabe. En effet, face au discours de l'Alya qui ne cesse d'invoquer une présence multiséculaire, les Arabes ont eu recours à une « remontée dans le temps » auparavant absente des référents communs, puisant pour l'essentiel leurs mythes originaires dans les racines musulmanes et dans l'Arabie du $7^{\mathrm{e}}$ siècle. L'archéologie, d'abord entreprise coloniale, fait défiler quatre ou cinq millénaires de passé supplémentaire, agrégés tardivement au lexique nationaliste.

Cette quête d'un passé pré-arabe, donnant un socle à l'attachement territorial, trouve aujourd'hui une expression dans la fabrication patrimoniale d'identités nationales au sens strict $^{4}$. Ainsi les régimes en place mettent-ils en scène le passé antique dans des musées modernes et prestigieux (Tunisie punique et romaine, Liban phénicien, Égypte pharaonique, etc.). Des lieux de mémoire se constituent, comme par exemple autour de la pêche perlière au Koweït, à Dubaï ou au Qatar.

(3) Voir l'article de Denis Charbit dans ce numéro.

(4) Cf. sur le site de Baalbeck, au Liban : Ussama Makdisi, « The "Rediscovery" of Baalbek : A Metaphor for Empire in the Nineteenth Century » et Jens Hanssen, « Imperial Discourses and an Ottoman Excavation in Lebanon », in Hélène Sader, Thomas Scheffler et Angelika Neuwirth (dir.), Baalbek : Image and Monument, 1898-1998, Beyrouth, Orient Institute, 1998 , p. $137-156,165-172$. 
Le patrimoine ottoman retrouve également peu à peu ses lettres de noblesse, à travers la restauration de bâtiments, la construction de mosquées nouvelles d'après un style « néo-ottoman », la représentation de cette période dans des films et feuilletons, etc. Cette dernière fabrication mémorielle semble en plus se réconcilier avec une unité arabe perdue, subsumée dans l'appartenance à l'Empire.

\section{À l'épreuve de la guerre, l'urgence culturelle}

La confrontation, non pas avec le sionisme comme idéologie nationale, mais avec Israël comme réalité, a ainsi créé un sentiment d'urgence culturelle. Ce sentiment affecte en premier lieu l'historiographie, bien souvent pensée en termes de « symétrie » ou de concurrence des mémoires. L'historienne Gudrun Krämer relève assez justement ceci, en regrettant que l'écriture de l'histoire de la Palestine soit affectée par une vision téléologique. Elle considère par exemple que le fait de marquer d'une pierre blanche la date de 1882 (date du décret ottoman autorisant l'achat de terre en Palestine pour les Juifs) est le résultat de ce regard rétrospectif. L'usage fait par la suite par l'État d'Israël de ces titres de propriété détermine la construction d'un « tournant historique » admis ${ }^{1}$.

Accompagnant le repli sur les frontières nationales strictes, il participe à définir de nouveaux points d'ancrage mémoriaux : fêtes, rites lieux. La communauté imaginée, ce sentiment d'appartenance, qu'il soit panarabe ou limité à un territoire plus restreint, est construit dans des pratiques qui ne se réduisent pas aux politiques étatiques, ni même au champ intellectuel et médiatique. Il se fonde aussi sur des lieux, des symboles inventés localement et par-

(I) Gudrun Krämer, A History of Palestine : From the Ottoman Conquest to the Founding of the State of Israël, Princeton, Princeton University Press, 2008. Voir le compte rendu dans ce numéro (p. 206-208). tagés par la communauté. En cela, la variation des échelles est le seul moyen de comprendre comment le nationalisme existe (ou n'existe pas) dans une région où coexistent des communautés différentes. Si le début du $20^{\mathrm{e}}$ siècle, et particulièrement à partir de la révolution jeune-turque, est le commencement de l'élaboration de doctrines nationalistes, il est aussi le moment de fermentation de pratiques communautaires, d'appropriations de symboles, de lieux, de saints. Les travaux d'un certain nombre de chercheurs, s'attachant à ces manifestations locales et aux déclinaisons multiples de la 'urûba, décrivent bien ces phénomènes ${ }^{2}$. Tous s'efforcent à retrouver le fil d'un temps long qui restitue à ces phénomènes une existence concrète, quotidienne. Ils contribuent également à redonner leur place à des acteurs souvent oubliés de ces études, et notamment les femmes (envisagées plus traditionnellement dans les études sur le nationalisme comme une « métaphore $»^{3}$ ). Il s'agit aussi de revenir aux lieux d'ancrage de la mémoire nationaliste, très vite « idéologisés » et souvent mal compris, de revenir aux acteurs et à la manière dont ils usent de ce nationalisme. Ainsi Eugene Rogan a-t-il pu montrer que le célèbre collège damascène Maktab Anbar, longtemps considéré comme le haut-lieu de développement du nationalisme arabe, devait plutôt être vu comme le lieu de construction d'une identité ottomanearabe $^{4}$. Il rejoint les pistes décrites il y a plus de vingt ans par Rashid Khalidi dans « Ottomanism and Arabism in Syria before 1914 : A

(2) Voir James Gelvin, op. cit. ; Emma Aubin-Boltanski, Pèlerinages et nationalisme en Palestine : prophètes, héros et ancêtres, Paris, Éd. de l'EHESS, 2008 ; Catherine Mayeur-Jaouen, Pèlerinages d'Égypte : histoire de la piété copte et musulmane, $X V^{e}-X X^{e}$ siècles, Paris, Éd. de l'EHESS, 2005.

(3) Voir Beth Baron, Egypt as a Woman : Nationalism, Gender, and Politics, Berkeley, University of California Press, 2005.

(4) Eugene Rogan, « Maktab Anbar », in Thomas Philipp et Christoph Schumann (dir.), From the Syrian Land to the States of Syria and Lebanon, Würzburg, Ergon, « Beiruter Texte und Studien, $96 \gg, 2004$. 
Reassessment $^{1}$ ». Déjà en 1991, les interrogations sur la postérité politique du nationalisme arabe stricto sensu poussaient les chercheurs à élaborer d'autres questionnements et d'autres grilles d'analyse sur la société de la fin de l'Empire ottoman ${ }^{2}$. L'analyse des discours et des parcours des acteurs montre bien que la naissance du nationalisme arabe s'est faite dans un plus grand «flou » qu'on ne le dit souvent : les appartenances (territoriales, religieuses, tribales) et les fidélités coexistent, hier comme aujourd'hui. Elles peuvent s'afficher dans les textes ou sur les murs des maisons ${ }^{3}$. Rendre aux acteurs leur liberté par rapport aux discours officiels signifie également comprendre en profondeur leurs stratégies et les rapports de force en présence qui font par exemple que l'islamisme aujourd'hui constitue une réponse jugée plus « efficace » que celle du simple nationalisme arabe, dépassé par ses échecs politiques.

Finalement, force est de constater que toute histoire du nationalisme arabe est nécessairement culturelle et travaillée par les évolutions politiques qui affectent la région. En dernier ressort, des identités, même particulières, se définissent sur la culture.

Alors, le nationalisme arabe tel qu'il persiste est probablement une exception, lui qui s'est appuyé sur une culture populaire, diffuse, et a marginalisé les efforts faits pour le transmuer en « culture officielle », probablement parce que cette culture, beaucoup trop coercitive, n'a pas tiré les leçons des échecs qui l'ont accompagnée. Le nationalisme arabe, sous sa forme la plus idéologique, demeure sous une forme atténuée, comme un levier pour « mobiliser » des citoyens arabes de plus en plus désabusés. La nation arabe, elle, se nourrit d'une culture de plus en plus mondialisée, en arabe, dans la diversité, mais elle est aussi, pour reprendre les mots d'Elias Khoury à propos de l'introuvable « rue arabe », « faite de toutes ces rues pleines de pauvres et de malheureux qui ne voient rien d'autre qu'une solution individuelle à leurs problèmes et qui cherchent sur les écrans une illusoire compensation ${ }^{4} \gg$.

Leyla Dakbli, Collège Gustave Flaubert, 75013, Paris, France.

Agrégée d'histoire et ancienne élève de l'École normale supérieure de Fontenay/Saint-Cloud, Leyla Dakhli est l'auteure d'une thèse de doctorat consacrée aux intellectuels syrolibanais à l'époque mandataire, soutenue en décembre 2003 à I'Université de Provence et récemment publiée aux Éditions IISMM-Karthala : Une génération d'intellectuels arabes, Syrie et Liban (1908-1940). (leyladakhli@hotmail.com)
(I) Dans Rashid Khalidi, Lisa Anderson, Muhammad Muslih et Reeva S. Simon (dir.), op. cit., p. 50-69.

(2) Voir l'article de Marc Aymes dans ce numéro.

(3) Voir Stefan Weber, « Images of Imagined Worlds : Self Image and Worldview in Late Ottoman Wall Paintings of Damascus », in Jens Hanssen, Thomas Philipp et Stephan Weber (dir.), The Empire in the City : Arab Provincial Capitals in the Late Ottoman Empire, Beyrouth, Orient Institute, « Beiruter Texte und Studien, $88 \gg, 2002$, p. 145-171.
(4) Elias Khoury, op. cit. 